\title{
Retrospective Validation of Prehospital Acute Stroke Severity Scale to Predict Large Vessel Occlusion in Acute Stroke Patients - Single Center study in China
}

Xiaoli Si ( $\sim$ sxl_20061@126.com )

Zhejiang University

Yuanjian Fang

Zhejiang university

Wenqing Xia

Zhejiang University

Tianwen Chen

Zhejiang university

Huan Huang

Zhejiang university

Keqin Liu

Zhejiang university

Lin Jiang

Zhejiang university

Congguo Yin

Zhejiang University

Research article

Keywords: emergent large vessel occlusion; prehospital stage; scales; stroke; endovascular therapy; triage

Posted Date: July 2nd, 2019

DOI: https://doi.org/10.21203/rs.2.10860/v1

License: (1) (1) This work is licensed under a Creative Commons Attribution 4.0 International License.

Read Full License 


\section{Abstract}

Background and Purpose - To date, identifying emergent large vessel occlusion (ELVO) patients in the prehospital stage is important but still challenging. We aimed to retrospectively validate a simple prehospital stroke scale--Prehospital Acute Stroke Severity (PASS) scale to identify ELVO. Methods - We retrospectively evaluated our consecutive cohort of acute ischemic stroke (AIS) who underwent CT angiography (CTA), MR angiography (MRA) or digital subtraction angiography (DSA). PASS scale was calculated based on National Institutes of Health Stroke Scale (NIHSS) items retrospectively. The comparison of diagnostic parameters between PASS scale and NIHSS scale were performed. Results Finally, a total of 605 patients were enrolled. ELVO patients with PASS $\geq 2$ had a median NIHSS score of 14. The best predictive value of PASS $\geq 2$ showed a similar predictive value compared with NIHSS $\geq 9$. Cortical symptoms such as consciousness disorder and gaze palsy were more specific indicators for ELVO than motor deficits. Consciousness disorder was more serious in posterior circulation infarct (PIC) while gaze palsy was more common in anterior circulation infarct (AIC). Conclusions - PASS scale had both good discrimination and calibration in our retrospective cohort. It could reflect acute stroke severity well and predict ELVO in an effective and simple way. Moreover, cortical symptoms had high specificities to predict ELVO on their own.

\section{Background}

Reperfusion with intravenous tissue plasminogen activator (recombinanttissue-typeplasminogenactivator, rt-PA) and endovascular therapy (EVT) improves outcome in patients with acute ischemic stroke (AIS) [1]. The efficiency of both treatments is highly time dependent [2]. Intravenous thrombolysis can be administered in Primary Stroke Centres (PSCs) while endovascular treatment can only be administered in EVT-capable centers in Comprehensive Stroke Centres (CSCs) [3]. Several recent studies have demonstrated that patients may have a better outcome by shorten the delay to EVT. Therefore, a simple and accurate assessment of emergent large vessel occlusion (ELVO) for primary paramedics transporting appropriate patients to an EVT-capable center is urgent.

Computed tomography angiography (CTA), magnetic resonance angiography (MRA) and digital subtraction angiography (DSA) allow the rapid assessment of vessel status in AIS. However, they are not broadly available 24 hours per day, particularly for PSCs with limited imaging resources [4]. Thus, surrogate markers of vessel occlusion will be helpful. The National Institutes of Health Stroke Scale (NIHSS) has a relatively strong relationship between neurological deficit and vessel status [5]. However, it is complex for emergency assessment and necessitating regular training for primary paramedics. There have been several prehospital stroke scales to identify patients experiencing ELVO [6-9]. Among them, Prehospital Acute Severity Scale (PASS) simplifies the parameters and indicates cortical symptoms more accurate [10].

Thus, the objective of this study is to retrospectively evaluate the predictive value of PASS scale on the detection of ELVO. We proposed that cortical symptoms-consciousness disorder and gaze palsy may be 
more sensitive indicators than motor deficits. Furthermore, we detailed the information on specific location of ELVO to validate the utility of PASS scale and NIHSS scale in different vessel status which is first reported.

\section{Methods}

Subjects and Methods

We retrospectively reviewed a historical cohort of 720 patients in our stroke unit from January 2016 to January 2018, Hangzhou first people's hospital, Zhejiang university. We enrolled patients who (1) were clinical suspicion of AIS (symptom onset $\leq 24$ hours, including patients who were intracerebral hemorrhage $(\mathrm{ICH})$ or with final nonvascular diagnosis like status epilepticus, syncope, metabolic disturbance and other reasons), (2) were examined by 2 experienced stroke neurologists and assessed the NIHSS scale at admission (the assesments of NIHSS scale were written on sheets).

PASS scale was calculated based on NIHSS scale retrospectively. This scale assessed 3 parameters: (1) level of consciousness [scored 0-1], (2) gaze [0-1], and (3) upper motor function [0-1]. Level of consciousness was classified as being disturbed with incorrect month and/or age.

ELVO was defined as occlusion of internal carotid artery (ICA), horizontal segment (M1) and insula segment (M2) of the middle cerebral artery (MCA), posterior cerebral artery (PCA), basilar artery (BA) and vertebral artery (VA) on CTA, MRA or DSA. Two experienced neurologists blinded to the patients' information assessed the occlusion on CTA, MRA or DSA with rater discrepancies settled by consensus. Basic patients characteristics were recorded in Table1. Patients with inconclusive or missing information on NIHSS or were not eligible for imaging assessment were excluded. Flowchart of the study population was in Figure 1. The study was approved by Zhejiang Provincial Natural Science Foundation of China (GF18H090036), and this study has required ethic approval.

\section{Statistical Analysis}

Patients were dichotomized into an ELVO group and a non-ELVO (NELVO) group. NELVO group included AIS patients without ELVO, ICH patients and with other final nonvascular diagnosis patients like status epilepticus, syncope, metabolic disturbance and other reasons. For statistical analysis, SPSS 10.0 software was used. Clinical characteristics were presented as the mean \pm standard deviation (SD) and as median (interquartile range, IQR), and differences between the two groups were estimated by the t-test or Mann-Whitney U-test if they were continuous variables. Categorical or binary datum was summarized by proportion ( $\mathrm{n} \%$ ); and differences between the two groups were estimated by the Pearson $\chi 2$ test. Receiver operating curves (ROC) and areas under receiver operating curve (AUC) were calculated as a measure of predictive ability for ELVO of the PASS and NIHSS scales, and for different items of PASS scales. The ROC derived optimal cutoff was determined at the maximal Youden Index. Hosmer-Lemeshow test was used to evaluate the calibration ability of PASS scales. Cross tables for different cutoff values of PASS 
scale were used to evaluate sensitivity, specificity, positive predictive value (PPV) and negative predictive value (NPV). Value with $p<0.05$ was regarded as significant.

\section{Results}

In total, we screened 720 patients in our stroke unit from January 2016 to January 2018. Finally, 605 patients were included (Table 1). Figure 1 summarized the flowchart of study population. In ELVO group, the mean age was $70 \pm 12$ years, males were $141(59 \%)$, the median time between symptom onset to assessment was $4.75 \pm 4.37$ hours, the median NIHSS score was 17 (interquartile range $=6.25$ ). In NELVO group, the mean age was $69 \pm 14$ years, males were 232 (63\%), the median time between symptom onset to assessment was $5.69 \pm 5.08$, the median NIHSS score was 3 (interquartile range $=5$ ) (Table 2).

Figure 2A showed the best cut point to predict ELVO was PASS $\geq 2$ while the best predictive value was NIHSS $\geq 9$. PASS $\geq 2$ showed AUC 0.89 (95\% Cl, 0.857-0.922), sensitivity $90.3 \%$, specificity $81.9 \%$, PPV $85.7 \%$ and NPV $11.9 \%$. NIHSS $\geq 9$ showed AUC 0.946 (95\% Cl, 0.924-0.968), sensitivity $94.1 \%$, specificity $84.9 \%$, PPV $85.1 \%$, and NPV 8.6\% (Figure 2A, Table 4). PASS $\geq 2$ showed a similar predictive value $($ Pearson $\chi 2$ test, chi2 $(1)=227.557, p<0.001)$ compared with NIHSS $\geq 9$ (Pearson $\chi 2$ test, chi2 $(1)=$ $250.317, p<0.001)$. ELVO patients identified with PASS $\geq 2$ had a median NIHSS score of 14 , while PASS $<2$ had a median NIHSS score of 4 (Table 3), which results were consistent with previous research [10]. Hosmer-Lemeshow test indicated there was no significant difference between predicted value and observed value (Hosmer-Lemeshow test, $X^{2}=4.046, p=0.853$ ) (Figure 2B).

Figure $2 \mathrm{C}$ showed the strongest predictor of ELVO through 3 parameters was consciousness disorder, with AUC $=0.794(95 \% \mathrm{Cl}, 0.750-0.837)$, sensitivity $74.8 \%$, specificity $83.9 \%$. The second strongest predictor was gaze palsy and/or deviation, with $\mathrm{AUC}=0.779(95 \% \mathrm{Cl}, 0.734-0.824)$, sensitivity $71.8 \%$, specificity $83.9 \%$. The arm weakness showed AUC $=0.645$ (95\% Cl, 0.592-0.698), sensitivity $71.8 \%$, specificity $83.9 \%$.

Table 4 showed the relationships between each clinical symptom, clinical scores and vessel occlusion types. PASS score in ACl was higher than PCl (Mann-Whitney U-test, $\mathrm{p}<0.001)$ while NIHSS score was higher in PCI (Mann-Whitney U-test, p=0.007). Detailed vessel occlusion typies were ACA 5 (3\%), CA 67 (34\%), MCA 124 (63\%), PCA 4 (2\%), BA 32 (14\%), VA 5 (2\%) (Table 4).

Figure 3 showed ELVO group had a higher percentage of consciousness disorder (Mann-Whitney U-test, $\mathrm{p}<0.001$ ) and gaze palsy than AIS-ELVO group (Mann-Whitney U-test, $\mathrm{p}<0.001$ ). ACl had a higher percentage of gaze palsy (Mann-Whitney U-test, $p<0.001$ ) and $\mathrm{PCl}$ a higher percentage of consciousness disorder (Mann-Whitney U-test, $p=0.003$ ).

Table 5 showed both PASS scale (Mann-Whitney U-test, $p=0.579$ ) and NIHSS score (Mann-Whitney U-test, $p<0.001)$ were higher in left hemisphere than right hemisphere (Mann-Whitney U-test, $p<0.001)$. Left hemisphere ELVO had a higher percentage of consciousness disorder (Mann-Whitney U-test, $p=0.036$ ) 
while right hemisphere ELVO had a higher percentage of gaze palsy (Mann-Whitney U-test, $p=0.051$ ) (Table 5, Figure 3C).

\section{Discussion}

To date, no triage strategy performs perfectly in identifying ELVO at prehospital. NIHSS scale has demonstrated to be predictive of ELVO, but prehospital assessment by medical emergency technicians may be difficult and time-consuming. And paramedics in PSCs may be inexperienced in the assessment of NIHSS scale. Moerover, patients with right hemispheric AIS may present mild to moderate symptoms which are underrepresented with NIHSS scale that right ELVO may be misdiagnosed. Previously an European group has designed and demonstrated PASS scale as a simple tool highly predicts ELVO with a suspicion AIS at prehospital. There are several advantages of PASS scale over preexisiting scales. First, PASS scale only includes consciousness disorder, gaze palsy and arm weakness, which parameters are simple but effective to evaluate. Second, emergency paramedics adopt level of consciousness questions is objective and could be combined with the evaluation of aphasia and dysarthria which many previous scales ignored[11, 12]. Besides, items in NIHSS scale range from normal to severe (0-4), which may be inaccurate due to the subjective judgment, as well as other scales like 3ISS, LAMS, RACE and so on[3, 9, 13-15]. While each parameter in PASS scale range from 0-1. Moreover, gaze palsy can be easily observed by paramedics rather than neglect or field of vision $[11,16]$. Last but not least, different form 3ISS, LAMS scales, PASS scale gives a higher weight to cortical symptoms rather than motor symptoms, which are typcial sighs of ELVO. Because motor symptoms can also occur in lacunar stroke and may not be good indicators for ELVO.

Stroke risks vary among ethnic populations, with much higher incidence and mortality reported in Asia. Recent research has revealed that Asian has a higher rate of large artery atherosclerotic stroke which may has a better collateral circulation than European who confuse with embolism [17]. PASS scale includes three parameters: consciousness disorder, gaze palsy and arm weakness. Consciousness disorder and gaze palsy are cortical symptoms which may manifest a poor collateral circulation. Thus, PASS scale may be unsuitable for Asian to evaluate ELVO. However, PASS scale in our retrospective cohort had both good discrimination and calibration ability, which results were consistent with previous research. Therefore, PASS scale can also be an effective and simple tool to detect ELVO patients for Asian.

There are several strengths in our study. First, our data included vessel typies of ELVO. The most type in both left and right hemisphere was MCA-ELVO. Second, the cohort included both $\mathrm{ACl}$ and $\mathrm{PCl}$ as opposed to some other scales just focused on ACl. Because of the poor prognosis of PCl, the utility of PASS scale may make important role at prehospital. In this cohort, $\mathrm{ACl}$ with more gaze palsy and $\mathrm{PCl}$ with more consciousness disorder. Third, as right hemispheric AIS may be misdiagnosed as its mild to moderate symptoms, we analysed different predictors in both hemispheres. We found PASS score in left ACl was higher than right and left $\mathrm{ACl}$ with more consciousness disorder while right $\mathrm{ACl}$ with more gaze palsy. Morover, this study indicated that the strongest predictor was consciousness disorder, than gaze palsy and/or deviation. Recently, gaze palsy and/or deviation has been the only parameter in an ELVO screen 
as an ideal prehospital scale [17]. Thus, we may further design a simpler and faster scale which just focus on cortical symptoms and validate prospectively in the field by trained paramedics prehospital in the future.

Our study has several limitations. First, this model was made from a single retrospective cohort. Perspective and large multicenter data would be more believable and compelling. Second, as a global scale with a limited range ( 0 to 3 ), PASS scale is insensitive to small differences between patients and to small changes in clinical status for individual patients. However, the complexity of NIHSS scale is responsible for its infrequent use in clinical routine. In contrast, the PASS scale is simple and quick to apply. Moreover, PASS score in ACl was higher than PCl, however, NIHSS score was higher in PCI than $\mathrm{ACl}$. The accuracy of PASS scale in PCI need further investigation and improvement.

\section{Conclusions}

In summary, we found PASS scale could be an easily memorized and effective tool to identify AIS with highly likelihood of ELVO and it had a similar accuracy with NIHSS. Additional studies concerning the utility of PASS scale in prehospital setting and its ability to predict stroke outcome are warranted. Further more, a simpler and faster scale focuses on cortical symptoms and validate prospectively in the field is urgent to yield in. Future studies that are in the prehospital setting will need to test scales while patient has not yet arrived to hospital, possibly to change destination triage decisions and transfer appropriate patients to a CSC quickly.

\section{Declarations}

\section{Acknowledgments}

Not Applicable.

\section{Author Contributions Statement}

$\mathrm{XS}$ and YF: contribute study design and draft the manuscript; $\mathrm{TC}$ and $\mathrm{HH}$ : collect patients' data; $\mathrm{KL}$ and WX: analyse patients' data; $L J$ and $C Y$ : revise the manuscript. All authors read and approved the final manuscript.

\section{Funding}

This work was supported by Zhejiang Provincial Natural Science Foundation of China (GF18H090036).

\section{The Availability of data and materials}

The datasets used and/or analysed during the current study available from the corresponding author on reasonable request. 


\section{Ethics approval and consent for participate}

We thank the patients and their relatives for their generous donation of samples. The consent we obtained from study participants was verbal and this was approved by the ethics committee. The study was approved by Zhejiang Provincial Natural Science Foundation of China (GF18H090036), and this study has required ethic approval.

\section{Conflict of Interest Statement}

The authors have no financial conflicts of interest.

\section{References}

[1] B.C.V. Campbell, G.A. Donnan, K.R. Lees, W. Hacke, P. Khatri, M.D. Hill, M. Goyal, P.J. Mitchell, J.L. Saver, H.C. Diener, S.M. Davis, Endovascular stent thrombectomy: the new standard of care for large vessel ischaemic stroke, Lancet Neurol, 14 (2015) 846-854.

[2] B.C. Campbell, A. Meretoja, G.A. Donnan, S.M. Davis, Twenty-Year History of the Evolution of Stroke Thrombolysis With Intravenous Alteplase to Reduce Long-Term Disability, Stroke, 46 (2015) 2341-2346.

[3] N. Perez de la Ossa, D. Carrera, M. Gorchs, M. Querol, M. Millan, M. Gomis, L. Dorado, E. Lopez-Cancio, M. Hernandez-Perez, V. Chicharro, X. Escalada, X. Jimenez, A. Davalos, Design and validation of a prehospital stroke scale to predict large arterial occlusion: the rapid arterial occlusion evaluation scale, Stroke, 45 (2014) 87-91.

[4] D.P. Briley, T. Meagher, D. King, Practical limitations of acute stroke MRI due to patient-related problems, Neurology, 64 (2005) 400-401; author reply 400-401.

[5] L. Derex, N. Nighoghossian, M. Hermier, P. Adeleine, J.C. Froment, P. Trouillas, Early detection of cerebral arterial occlusion on magnetic resonance angiography: predictive value of the baseline NIHSS score and impact on neurological outcome, Cerebrovasc Dis, 13 (2002) 225-229.

[6] C.S. Kidwell, S. Starkman, M. Eckstein, K. Weems, J.L. Saver, Identifying stroke in the field. Prospective validation of the Los Angeles prehospital stroke screen (LAPSS), Stroke, 31 (2000) 71-76.

[7] D.L. Tirschwell, W.T. Longstreth, Jr., K.J. Becker, R.E. Gammans, Sr., L.A. Sabounjian, S. Hamilton, L.B. Morgenstern, Shortening the NIH Stroke scale for use in the prehospital setting, Stroke, 33 (2002) 28012806.

[8] J.N. Llanes, C.S. Kidwell, S. Starkman, M.C. Leary, M. Eckstein, J.L. Saver, The Los Angeles Motor Scale (LAMS): a new measure to characterize stroke severity in the field, Prehosp Emerg Care, 8 (2004) 46-50.

[9] L.A. Beume, M. Hieber, C.P. Kaller, K. Nitschke, J. Bardutzky, H. Urbach, C. Weiller, M. Rijntjes, Large Vessel Occlusion in Acute Stroke, Stroke, 49 (2018) 2323-2329. 
[10] S. Hastrup, D. Damgaard, S.P. Johnsen, G. Andersen, Prehospital Acute Stroke Severity Scale to Predict Large Artery Occlusion: Design and Comparison With Other Scales, Stroke, 47 (2016) 1772-1776.

[11] F.O. Lima, G.S. Silva, K.L. Furie, M.R. Frankel, M.H. Lev, E.C. Camargo, D.C. Haussen, A.B. Singhal, W.J. Koroshetz, W.S. Smith, R.G. Nogueira, Field Assessment Stroke Triage for Emergency Destination: A Simple and Accurate Prehospital Scale to Detect Large Vessel Occlusion Strokes, Stroke, 47 (2016) 19972002.

[12] B.S. Katz, J.T. McMullan, H. Sucharew, O. Adeoye, J.P. Broderick, Design and validation of a prehospital scale to predict stroke severity: Cincinnati Prehospital Stroke Severity Scale, Stroke, 46 (2015) 1508-1512.

[13] U. Fischer, M. Arnold, K. Nedeltchev, C. Brekenfeld, P. Ballinari, L. Remonda, G. Schroth, H.P. Mattle, NIHSS score and arteriographic findings in acute ischemic stroke, Stroke, 36 (2005) 2121-2125.

[14] M.R. Heldner, C. Zubler, H.P. Mattle, G. Schroth, A. Weck, M.L. Mono, J. Gralla, S. Jung, M. El-Koussy, R. Ludi, X. Yan, M. Arnold, C. Ozdoba, P. Mordasini, U. Fischer, National Institutes of Health stroke scale score and vessel occlusion in 2152 patients with acute ischemic stroke, Stroke, 44 (2013) 1153-1157.

[15] R.L. Maclsaac, M. Ali, M. Taylor-Rowan, H. Rodgers, K.R. Lees, T.J. Quinn, V. Collaborators, Use of a 3Item Short-Form Version of the Barthel Index for Use in Stroke: Systematic Review and External Validation, Stroke, 48 (2017) 618-623.

[16] M.S. Teleb, A. Ver Hage, J. Carter, M.V. Jayaraman, R.A. McTaggart, Stroke vision, aphasia, neglect (VAN) assessment-a novel emergent large vessel occlusion screening tool: pilot study and comparison with current clinical severity indices, J Neurointerv Surg, 9 (2017) 122-126.

[17] R.V. Krishnamurthi, V.L. Feigin, M.H. Forouzanfar, G.A. Mensah, M. Connor, D.A. Bennett, A.E. Moran, R.L. Sacco, L.M. Anderson, T. Truelsen, M. O'Donnell, N. Venketasubramanian, S. Barker-Collo, C.M. Lawes, W. Wang, Y. Shinohara, E. Witt, M. Ezzati, M. Naghavi, C. Murray, I.R.F.S. Global Burden of Diseases, G.B.D.S.E. Group, Global and regional burden of first-ever ischaemic and haemorrhagic stroke during 1990-2010: findings from the Global Burden of Disease Study 2010, Lancet Glob Health, 1 (2013) e259-281.

\section{Tables}

Table 1. Baseline demographic and clinical characteristics of participants $(n=605)$ 


\begin{tabular}{|c|c|}
\hline Patient characteristics & No(\%) \\
\hline Sex, male & $369(61.0)$ \\
\hline Median age, years (mean \pm SD) & $70 \pm 12$ \\
\hline Time symptom onset to assessment, hour (mean \pm SD) & $5.25 \pm 5.08$ \\
\hline ELVO & $237(39.2)$ \\
\hline NELVO & $368(60.8)$ \\
\hline AIS-NELVO & $199(54.1)$ \\
\hline $\mathrm{ICH}$ & $103(28.0)$ \\
\hline Stroke mimic* & $66(17.9)$ \\
\hline
\end{tabular}

SD, standard deviation; No, number; ELVO, emergent large vessel occlusion; NELVO, non-emergent large vessel occlusion; ICH, intracerebral hemorrhage;

*Stroke mimic included status epilepticus $(n=23)$, syncope $(n=3)$, metabolic disturbance $(n=15)$, and other reasons $(n=25)$.

Table 2. Demographic and clinical characteristics in different groups $(n=605)$

\begin{tabular}{|c|c|c|}
\hline Patient characteristics & ELVO & NELVO \\
\hline Patient number (no, \%) & $237(39.2)$ & $368(60.8)$ \\
\hline Sex, male (no, \%) $\square$ & $141(59.5)$ & $232(63.0)$ \\
\hline Age, years (mean $\pm \mathrm{SD}$ ) & $70 \pm 12$ & $69 \pm 14$ \\
\hline Time symptom onset to assessment, hour (mean \pm SD) & $4.75 \pm 4.37$ & $5.69 \pm 5.08$ \\
\hline NIHSS median (IQR) & $17(6.25)$ & $3(5)$ \\
\hline ACI in AIS (no, \%) & $196(82.7)$ & $163(44.3)$ \\
\hline Left hemisphere/ACI in AIS (no, \%) & $98(50)$ & $92(56.4)$ \\
\hline
\end{tabular}

ELVO, emergent large vessel occlusion; NELVO, non-emergent large vessel occlusion; SD, standard deviation; no, number; NIHSS, National Institutes of Health Stroke Scale; IQR, interquartile range; ACI, Anterior circulation infarct.

Table 3. Sensitivity, Specificity, PPV, NPV, and Overall Accuracy of Different Cutoff Values of the PASS for the Detection of ELVO 
\begin{tabular}{lllllllll}
\hline PASS & Total no. & ELVO no. & Sensitivity (\%) & Specificity (\%) & PPV (\%) & NPV (\%) & Accuracy (\%) & NIHSS
\end{tabular}

\begin{tabular}{ccccccccc}
\hline$\geq 1$ & 294 & 236 & 99.6 & 29.1 & 80.3 & 0.7 & 80.3 & 4 \\
$\geq 2$ & 251 & 215 & 90.3 & 81.9 & 85.7 & 11.9 & 85.7 & 14 \\
3 & 140 & 126 & 53.4 & 93 & 90 & 37.5 & 90 & 18 \\
\hline
\end{tabular}

PPV, positive predictive value; NPV, negative predictive value.

Table 4. Association between clinical symptoms, scores and vessel occlusion types

\begin{tabular}{|c|c|c|c|}
\hline ELVO type & No (\%) & $\begin{array}{c}\text { PASS } \\
(\text { mean } \pm \text { SD) }\end{array}$ & $\begin{array}{l}\text { NIHSS } \\
\text { (IQR) }\end{array}$ \\
\hline AIS-NELVO & $199(45.6)$ & $0.96 \pm 0.06$ & $3(5)$ \\
\hline ELVO & $237(54.4)$ & $2.43 \pm 0.04$ & $17(7.75)$ \\
\hline $\mathrm{ACI}$ & $196(82.7)$ & $2.48 \pm 0.69$ & $16(7)$ \\
\hline ACA & $5(2.6)$ & & \\
\hline CA & 67 (34.2) & & \\
\hline MCA & $124(63.3)$ & & \\
\hline M1 & $100(80.6)$ & & \\
\hline M2 & 24 (19.4) & & \\
\hline PCI & $41(17.3)$ & $2.12 \pm 0.60$ & $22(5.5)$ \\
\hline PCA & $4(9.8)$ & & \\
\hline BA & 32 (78.0) & & \\
\hline VA & $5(12.2)$ & & \\
\hline Total & 436 & $1.76 \pm 1.05$ & $11(5)$ \\
\hline
\end{tabular}

ACA, anterior cerebral artery; CA, carotid artery; MCA, middle cerebral artery; M1, M1 segment; M2, M2 segment; PCA, posterior cerebral artery; BA, basilar artery; VA, vertebral artery.

Table 5. Association between clinical symptoms, scores and vessel occlusion types on left and right hemisphere 


\begin{tabular}{llcc}
\hline Hemisphere ELVO & No. (\%) & PASS & NIHSS \\
& & $($ mean \pm SD) & $($ IQR) \\
\hline Left ACI & $98(50)$ & $2.52 \pm 0.72$ & $18(6)$ \\
ACA & $1(1)$ & & \\
CA & $31(32)$ & & \\
MCA & $66(67)$ & & \\
M1 & $49(74)$ & & \\
M2 & $17(26)$ & & \\
Right ACI & $98(50)$ & $2.46 \pm 0.63$ & $14(5)$ \\
ACA & $4(1)$ & & \\
CA & $36(32)$ & & \\
MCA & $58(67)$ & & \\
M1 & $51(74)$ & & \\
M2 & $7(26)$ & & \\
\hline
\end{tabular}

ELVO, large vessel occlusion; NELVO, not large vessel occlusion; PASS, Prehospital Acute Stroke Severity Scale; NIHSS, National Institutes of Health Stroke Scale; IQR, interquartile range; ACA, anterior cerebral artery; CA, carotid artery; MCA, middle cerebral artery; M1, M1 segment; M2, M2 segment; PCA, posterior cerebral artery; BA, basilar artery; VA, vertebral artery.

\section{Figures}

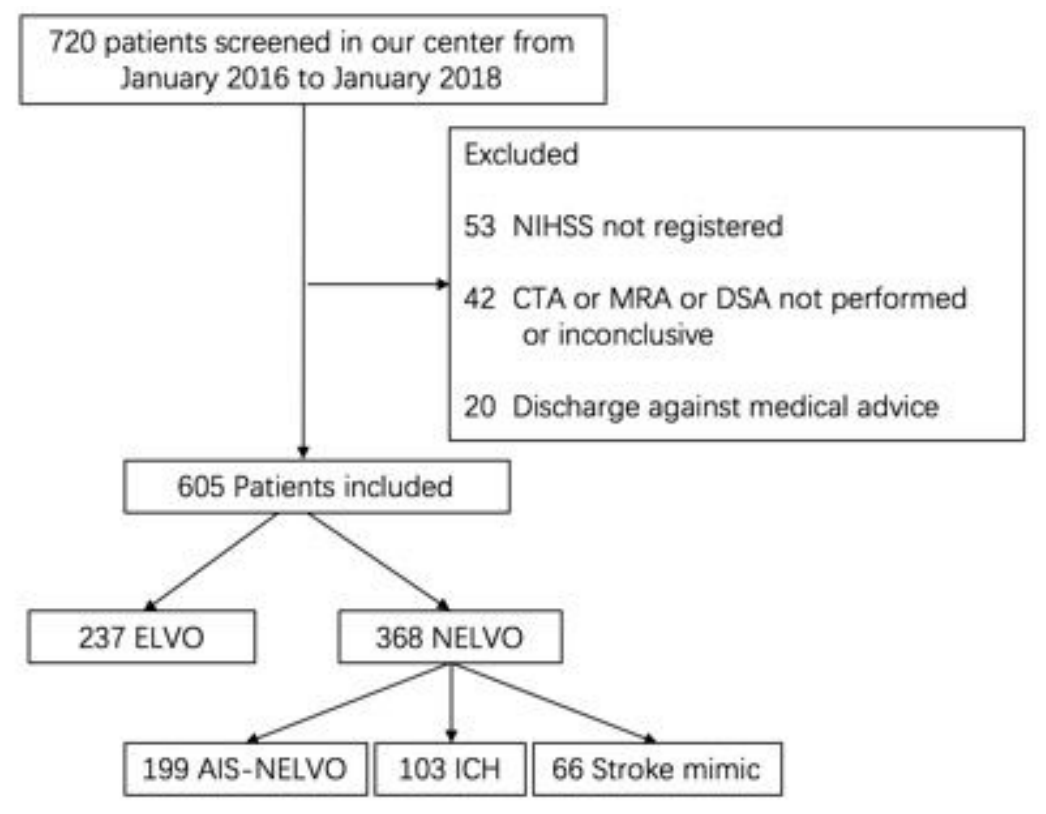

Figure 1

Flowchart of the the study population. NIHSS, National Institutes of Health Stroke Scale; CTA, computed tomography angiography; MRA, magnetic resonance angiography; DSA, digital substraction angiography; 
AIS, acute ischemic stroke; N-AIS, non-acute ischemic stroke; ELVO, emergent large vessel occlusion; NELVO, not emergent large vessel occlusion; ICH, intracerebral hemorrhage; EVT, endovascular therapy; NEVT, non-endovascular therapy.
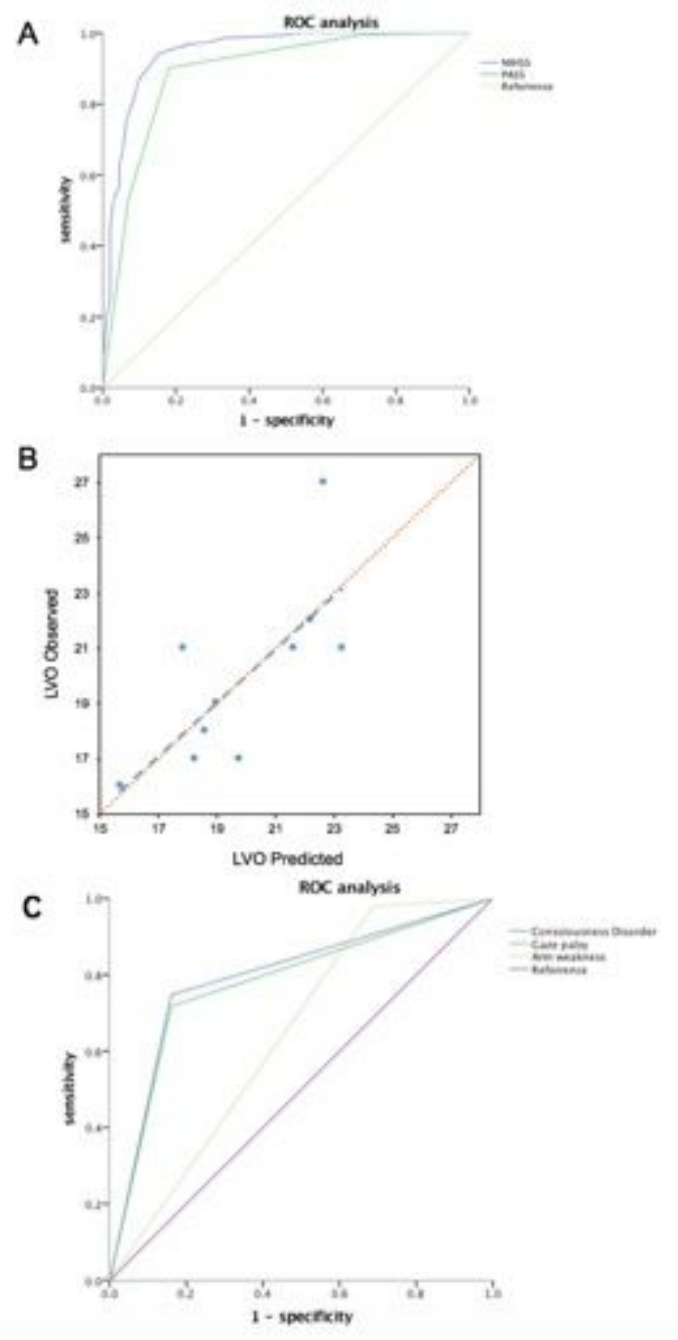

\section{Figure 2}

Discrimination and calibration analysis: A: PASS $\geq 2$ showed AUC 0.89 , sensitivity $90.3 \%$, specificity $81.9 \%$. NIHSS $\geq 9$ showed AUC 0.946 , sensitivity $94.1 \%$, specificity $84.9 \%$. B: There was no significant difference between predicted value and observed value. C: Consciousness disorder showed AUC $=0.794$, sensitivity $74.8 \%$, specificity $83.9 \%$; gaze palsy and/or deviation showed AUC $=0.779$, sensitivity $71.8 \%$, specificity $83.9 \%$; arm weakness showed AUC $=0.645$, sensitivity $71.8 \%$, specificity $83.9 \%$. 
A

Associatine between AIS yosel types and clinical symploms

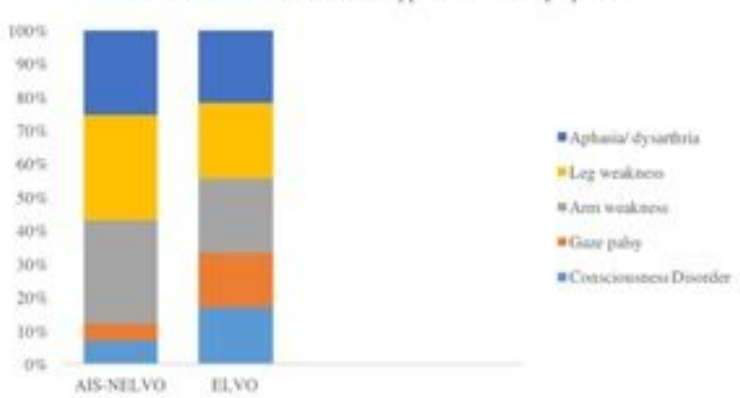

B

Association between EV VO vessel eoclosion types and clinical symploms

1005

ops

$\operatorname{sos}$

704

$\operatorname{ses}$

4005

305

然

os

MC

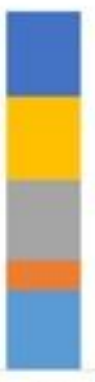

- Aptaner dicutaria

vLeg wedinces

- Amr vealines

sGave paley

acenowiveneses Diveter

C

Association between ELVO vessel occlusion types and clinical symptoms

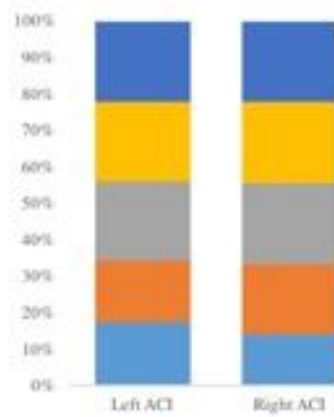

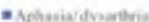

whoveainew

ofmerme

- Caarcpaloy

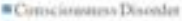

Figure 3

A: ELVO group had a higher percentage of consciousness disorder and gaze palsy than AIS-ELVO group. $\mathrm{B}$ : $\mathrm{ACl}$ had a higher percentage of gaze palsy and $\mathrm{PCl}$ a higher percentage of consciousness disorder. C: Left hemisphere ELVO had a higher percentage of consciousness disorder while right hemisphere ELVO had a higher percentage of gaze palsy. 